\title{
Transversity parton distribution functions from lattice QCD
}

\author{
Constantia Alexandrou, ${ }^{1,2}$ Krzysztof Cichy, ${ }^{3}$ Martha Constantinou, ${ }^{4}$ \\ Karl Jansen, ${ }^{5}$ Aurora Scapellato, ${ }^{1,6}$ and Fernanda Steffens ${ }^{7}$ \\ ${ }^{1}$ Department of Physics, University of Cyprus, P.O. Box 20537, 1678 Nicosia, Cyprus \\ ${ }^{2}$ Computation-based Science and Technology Research Center, \\ The Cyprus Institute, 20 Kavafi Street, Nicosia 2121, Cyprus \\ ${ }^{3}$ Faculty of Physics, Adam Mickiewicz University, Umultowska 85, 61-614 Poznań, Poland \\ ${ }^{4}$ Department of Physics, Temple University, Philadelphia, Pennsylvania 19122-1801, USA \\ ${ }^{5}$ NIC, DESY, Platanenallee 6, D-15738 Zeuthen, Germany \\ ${ }^{6}$ University of Wuppertal, Gaußstr. 20, 42119 Wuppertal, Germany \\ ${ }^{7}$ Institut für Strahlen-und Kernphysik, Rheinische Friedrich-Wilhelms-Universität Bonn, \\ Nussallee 14-16, 53115 Bonn, Germany
}

(Received 6 July 2018; published 26 November 2018)

\begin{abstract}
We present the first direct calculation of the renormalized transversity parton distribution function within the nucleon from lattice QCD. The calculation is performed using simulations with the light quark mass fixed to its physical value and at one value of the lattice spacing. Apart from physically light quarks, novel elements of the calculations are nonperturbative renormalization and extraction of a formula for the matching to lightcone parton distribution functions. Final results are presented in the $\overline{\mathrm{MS}}$ scheme at a scale of $\sqrt{2} \mathrm{GeV}$.
\end{abstract}

DOI: $10.1103 /$ PhysRevD.98.091503

\section{INTRODUCTION}

The proton is the simplest baryon. The strong force which is governing the internal dynamics of baryons makes them immensely complicated systems even already for the proton as the simplest baryon, with several nontrivial structure functions that are actively being investigated both theoretically and experimentally. One of the most important tools to understand QCD dynamics in a hadron are parton distribution functions (PDFs), which give information on the hadron spin and momentum distribution among its constituent quarks and gluons. During the past four decades, detailed information on PDFs has been accumulated as a function of the longitudinal momentum fraction $x$ of the nucleon momentum that they carry [1-3]. These encompass the class of chiral-even distributions: $f_{1}(x)$, for unpolarized partons, and $g_{1}(x)$, for helicity PDF in a longitudinally polarized nucleon. The remaining collinear transversity PDF, $h_{1}(x)$, which is defined as the difference between probabilities to find a parton spin aligned and antialigned to the transversely polarized parent nucleon, is considerably less known, because it is chiral odd $[4,5]$. This requires $h_{1}(x)$ to couple to another chiral-odd function in order to be accessed by experiments, excluding the traditional totally inclusive processes that have successfully

Published by the American Physical Society under the terms of the Creative Commons Attribution 4.0 International license. Further distribution of this work must maintain attribution to the author(s) and the published article's title, journal citation, and DOI. Funded by SCOAP ${ }^{3}$. unveiled different facets of the inner structure of the nucleon.

The transverse momentum dependent (TMD) Collins fragmentation function (FF) $H_{1}^{\perp}\left(x, p_{\perp}\right)$ [6] is chiral odd and couples to the TMD transversity $h_{1}\left(x, k_{\perp}^{2}\right)$ in the Collins single-spin asymmetry, which also contains information on the spin-averaged TMD FF and unpolarized TMD PDF. The FFs can be obtained independently from $e^{+} e^{-}$annihilation into dihadrons of transverse momentum $p_{\perp}$, with data available from BELLE [7,8], BABAR [9], and BESIII [9] collaborations. In conjunction with HERMES [10] and COMPASS [11,12], semi-inclusive deep-inelastic scattering (SIDIS) TMD data for single hadron production, $h_{1}\left(x, k_{\perp}^{2}\right)$, are then extracted. An ansatz [13] is used to disentangle the dependence on the momentum fraction from the transverse momentum on both the TMD FFs and the TMD PDFs to, finally, obtain the $h_{1}(x)$ distribution. Alternatively, in order to avoid the use of TMDs and to benefit from collinear factorization, it has been advocated [14-16] to use dihadron SIDIS cross section data to relate the measured asymmetry directly to $h_{1}(x)$. This asymmetry, integrated over the transverse momentum of the hadrons, is proportional to a simple product of $h_{1}(x)$ and an integral of the chiral-odd dihadron FF. The disadvantage of this route, as compared to the former case that uses single hadron production, is that the available data are less precise. In addition, collinear factorization is problematic at large $x$ [17]. Nevertheless, both procedures have been recently employed in global analyses for the extraction of the $x$-dependence of the nucleon transversity [18-23]. 
It is clear from this discussion that the extraction of the transversity distributions is sensitive to the FFs, with or without TMDs, and also to the TMD PDFs. Accordingly, reliable and independent information on $h_{1}(x)$ is important in its own right, as the computation of the $x$-dependence of PDFs from first principles is a milestone in itself. In addition to that, it has the potential to constrain the FFs, TMDs FFs, and TMDs PDFs. It is also clear from the Monte Carlo analysis of global data of Ref. [22] that SIDIS data by themselves do not impose tight constraints either to the $x$-dependence of the distributions or to the tensor charges associated with them (see, e.g., SIDIS data in Fig. 3). The isovector tensor charge, $g_{T}=\int_{0}^{1}\left(h_{1}^{u}(x)-h_{1}^{d}(x)\right) d x$, in particular, is found to be $0.9(8)$ when only SIDIS data are used. The inclusion of lattice data for $g_{T}$ in the global fit, on the other hand, moves $g_{T}$ to 1.0(1), while reducing the uncertainty in the corresponding $x$-dependence of the distributions by a factor of at least 3 , showing the enormous impact that information from first principles has on the determination of the transversity distributions. The analysis of Ref. [23], which uses dihadron interference FFs in SIDIS for a single-fit analysis of electron-proton and proton-proton data, however, has found that the individual $u$ and $d$ charges are incompatible between their results and those of Ref. [22]. A further comparison between the charges obtained from phenomenology and the ones from lattice QCD shows additional tension [24], reinforcing the idea of a transverse spin puzzle. Given that the tensor charge is a candidate to constrain physics beyond the Standard Model [25-28], it follows that a direct computation of the $x$-dependence of $h_{1}(x)$ has an impact in different branches of particle and nuclear physics from imposing limits on the hadron momentum fraction $x$ and the transverse momentum $p_{\perp}$-dependence of the FFs, to constraints on the extensions of the Standard Model itself. The overarching importance of transversity is translated into the inclusion of experiments aimed at measuring transversity in the thomas jefferson laboratory $12 \mathrm{GeV}$ program [24,29] and at the future Electron Ion Collider $[30,31]$, which will explore the large and small $x$ regions of $h_{1}(x)$, respectively.

As mentioned above, the PDFs extracted from experimental data use input from theoretically motivated parametrizations. However, it would be imperative to have prediction from first principles, and an ideal formulation is lattice QCD. In this paper, we present the first ab initio computation of the $x$-dependence for the isovector transversity PDF in lattice QCD, including a newly developed renormalization and matching procedure. This, together with simulating at the physical point, presents a major development as compared to previous studies $[32,33]$.

\section{QUASI-PDFS}

Light-cone PDFs cannot be directly evaluated on a Euclidean lattice, but certain information is obtained from their moments in lattice QCD (see e.g., Ref. [34-40] and references therein). The reconstruction of light-cone PDFs from their moments, however, is a difficult task, due to increasing statistical noise for high moments as well as due to a power divergence for moments with more than three covariant derivatives. Recently, a new direction has been proposed by X. Ji [41] to obtain the Bjorken- $x$ dependence of PDFs using lattice QCD, the so-called quasi-PDFs, defined for the transversity case as

$$
\tilde{h}_{1}(x, \Lambda, P)=\int_{-\infty}^{+\infty} \frac{d z}{4 \pi} e^{-i x P_{3} z} M_{h_{1}}(P, z) .
$$

$\Lambda \sim 1 / a$ is a UV cutoff, and $M_{h_{1}}(P, z)$ is the nonlocal matrix element

$$
\begin{gathered}
M_{h_{1}}(P, z)=\langle N(P)|\mathcal{O}(z)| N(P)\rangle, \\
\mathcal{O}(z)=\bar{\psi}(z) \frac{\sigma_{31}+\sigma_{32}}{2} W(z ; 0) \psi(0),
\end{gathered}
$$

between two nucleon states $|N\rangle$ having a spatial momentum $\vec{P}=\left(0,0, P_{3}\right)$, if the boost is in the $z$-direction. The Wilson line is taken along the direction of the momentum and has a length varying from zero up to the half of the spatial extent of the lattice. It connects a quark $(\psi)$ and antiquark $(\bar{\psi})$ with a Dirac matrix $\sigma_{3 i}$ in the transversity case, where the second index denotes the spatial direction of the nucleon spin, perpendicular to the direction of the momentum. For this particular choice of the Dirac structure, $M_{h_{1}}(P, z)$ does not exhibit mixing with other operators [42]. Note that each of the terms of Eq. (3) is projected appropriately to avoid statistical noise contamination. To get from quasi-PDFs to light-cone PDFs, bare matrix elements are renormalized, and then matching and target mass corrections (TMCs) are used, provided that nucleon momenta are large enough for Large Momentum Effective Theory to be applicable $[41,43]$. State-of-the-art results on the spin-averaged and helicity PDFs using the same setup appear in Ref. [44]. Results on these quantities were also presented in Ref. [45]. Alternative approaches for computing PDFs in lattice QCD were introduced and investigated in Refs. [46-50].

\section{NUMERICAL SETUP}

We use one ensemble of gauge configurations, produced with the Iwasaki-improved gauge action [51,52] and two degenerate $\left(N_{f}=2\right)$ twisted mass clover-improved fermions $[53,54]$ with their masses fixed approximately to their physical value [55]. The ensemble has a lattice volume of $48^{3} \times 96$, lattice spacing $a \simeq 0.093 \mathrm{fm}$, resulting in a spatial extent of $L \simeq 4.5 \mathrm{fm}$, and a pion mass $m_{\pi} \simeq 130 \mathrm{MeV}$.

In this work, we focus on the isovector flavor combination, $h_{1}^{u-d}$, that receives only connected contributions. We also obtain $h_{1}^{\bar{d}}(x)-h_{1}^{\bar{u}}(x)$ given the crossing relation $h_{1}^{\bar{q}}(x)=-h_{1}^{q}(-x)$ [56]. Lattice data are extracted from a 
ratio of $M_{h_{1}}(P, z)$ projected at zero momentum transfer, over the corresponding two-point function.

A state with the quantum numbers of the nucleon is created at a source $(t=0)$, annihilated at the sink $\left(T_{\text {sink }}\right)$, and couples with $\mathcal{O}$ at time slice $t_{\text {ins }}$. For sufficiently large time separations, $T_{\text {sink }}$, the desired matrix element of the nucleon ground state is then extracted. To improve the overlap with the ground state, we use Gaussian smeared interpolating fields $[57,58]$ with APE-smeared gauge links [59]. The three-point functions require the computation of an all-to-all propagator from all positions of the sink to all spatial insertion points and are evaluated by performing sequential inversions through the sink [60]. Moreover, to increase the overlap of the nucleon interpolating field with the ground state of the boosted particle, we use the momentum smearing technique [61], which has also been applied in our previous quasi-PDFs calculations [33,44]. For each value of the nucleon momentum used in this work, we tune the parameter entering the momentum smearing function in order to maximize the overlap with the boosted nucleon. This results in a significant reduction of the statistical uncertainties on the nucleon correlators. QuasiPDFs have been computed for three values of the nucleon momentum, namely, $P=\frac{6 \pi}{L}, \frac{8 \pi}{L}, \frac{10 \pi}{L} \quad(\sim 0.83,1.11$, $1.38 \mathrm{GeV}$ ) with $T_{\text {sink }}$ set to $12 a \simeq 1.12 \mathrm{fm}$. To keep the statistical errors approximately the same for the different boosts, we increase the number of configurations, as can be seen in Table I. For each configuration, we use multiple random source positions, and for $P=\frac{8 \pi}{L}, \frac{10 \pi}{L}$, we employ the truncated solver method [62] to reduce the computational cost. For each source position, we average over correlation functions where the momentum and Wilson line are aligned along all possible directions (namely, $\pm x, \pm y$, $\pm z$ ), to increase the number of measurements. To reduce statistical uncertainties in the renormalized $M_{h_{1}}(P, z)$, we apply five steps of three-dimensional stout smearing [63] to the gauge links entering the operator $\mathcal{O}$. We find no dependence of the renormalized $M_{h_{1}}(P, z)$ on the number of iterations as discussed in Ref. [44]. In Fig. 1, we show the momentum dependence of the bare $M_{h_{1}}(P, z)$. As expected, for higher momentum boosts, they decay to zero faster with increasing $z$, and the imaginary part becomes more prominent.

We examine ground state dominance on each momentum as we vary $T_{\text {sink. }}$. We use three values for $T_{\text {sink }}$, namely, $8 a$, $10 a$, and $12 a$. Convergence is seen between $T_{\text {sink }}=10 a$

TABLE I. Statistics used in this calculation for each momentum. $N_{\text {conf }}$ is the number of configurations, and $N_{\text {meas }}$ is the total number of measurements.

\begin{tabular}{lccc}
\hline \hline & $\frac{6 \pi}{L}(0.83 \mathrm{GeV})$ & $\frac{8 \pi}{L}(1.11 \mathrm{GeV})$ & $\frac{10 \pi}{L}(1.38 \mathrm{GeV})$ \\
\hline$N_{\text {conf }}$ & 100 & 425 & 811 \\
$N_{\text {meas }}$ & 9600 & 38250 & 72990 \\
\hline \hline
\end{tabular}

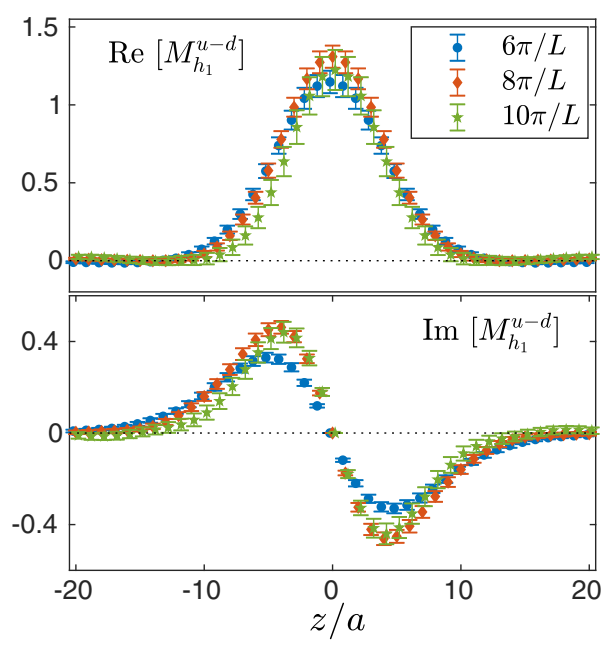

FIG. 1. Real (upper) and imaginary (lower) parts of the bare $M_{h_{1}}(P, z)$ at five stout steps, for $P=\frac{6 \pi}{L}$ (blue), $P=\frac{8 \pi}{L}$ (red), and $P=\frac{10 \pi}{L}$ (green), as a function of the Wilson line length, $z / a$.

and $T_{\text {sink }}=12 a$, and therefore we conclude that groundstate dominance is achieved for $T_{\text {sink }}=12 a$. In particular, the real part of Eq. (2) is compatible between $T_{\text {sink }}=10 a$ and $T_{\text {sink }}=12 a$, while the imaginary part in the small- $z$ region is more influenced by excited states. Note, however, that boosting the nucleon with momenta higher than what we presently use is expected to require even larger values of $T_{\text {sink }}$ to suppress excited states. For the momenta we employ, we have also checked the dispersion relation as a test of cutoff effects, and no deviations from the continuum energy-momentum dependence are observed.

\section{TOWARDS LIGHT-CONE PDFS}

The matrix elements of Eq. (2) have the additional complication of a powerlike divergence of the form $|z| / a$ inherited by the Wilson line. The latter, together with logarithmic divergences, must be eliminated prior to taking the Fourier transform of Eq. (1). Recently, we proposed a nonperturbative $\mathrm{RI}^{\prime}$-type prescription [64] that eliminates all divergences in a consistent and reliable fashion (see also Ref. [65] for an alternative prescription). The renormalization procedure is outlined in Ref. [64], and we use the same setup as in Ref. [44]. Using the results of Ref. [42], we convert the renormalization functions to the $\overline{\mathrm{MS}}$ scheme and evolve to $\mu=\sqrt{2} \mathrm{GeV}$, to match the scale used in the phenomenological data [22]. A linear extrapolation $\left[\left(a \mu_{0}\right)^{2} \rightarrow 0\right]$ is applied to eliminate residual dependence on the RI' scale $\mu_{0}$.

For a last step, we extract the renormalized light-cone PDF, $h_{1}(x, \mu)$, from renormalized quasi-PDF, $\tilde{h}_{1}\left(x, \mu, P_{3}\right)$, via a matching procedure [66-71],

$$
h_{1}(x, \mu)=\int_{-\infty}^{\infty} \frac{d \xi}{|\xi|} \delta C\left(\xi, \frac{\xi \mu}{x P_{3}}\right) \tilde{h}_{1}\left(\frac{x}{\xi}, \mu, P_{3}\right),
$$


where $\delta C\left(\xi, \frac{\xi \mu}{x P_{3}}\right)$ is the matching kernel. $\delta C$ can be computed perturbatively within QCD, because the IR physics is the same for quasi- and light-cone PDFs. $\delta C$ has been computed at one-loop order for the transversity case [66], using a hard transverse momentum cutoff and a quark mass to regularize the UV and the IR divergences, respectively. Here, we perform a computation using a gluon mass to regularize the IR divergences and dimensional regularization to regularize the UV divergences. The resulting kernel is independent of the gluon mass and takes quasi-PDFs renormalized in the $\overline{\mathrm{MS}}$ scheme to lightcone PDFs in this scheme. It reads

$$
\delta C\left(\xi, \frac{\xi \mu}{x P_{3}}\right)=\delta(1-\xi)+\frac{\alpha_{s}}{2 \pi} C_{F} \begin{cases}{\left[\frac{2 \xi}{1-\xi} \ln \frac{\xi}{\xi-1}+\frac{2}{\xi}\right]_{+}} & \xi>1, \\ {\left[\frac{2 \xi}{1-\xi}\left(\ln \frac{4 x^{2} P_{3}^{2}}{\xi^{2} \mu^{2}}+\ln (\xi(1-\xi))\right)-\frac{2 \xi}{1-\xi}\right]_{+}} & 0<\xi<1, \\ {\left[-\frac{2 \xi}{1-\xi} \ln \frac{\xi}{\xi-1}+\frac{2}{1-\xi}\right]_{+}} & \xi<0 .\end{cases}
$$

The plus prescriptions in Eq. (5) are all at $\xi=1$. The contributions outside the physical region in Eq. (5) have their origin exclusively in the one-loop correction to quasiPDFs. Conversely, quasi-PDFs are UV finite inside the physical region. Thus, unlike light-cone PDFs, the UV divergence in quasi-PDFs appears when integrating the momentum fraction in the one-loop wave-function correction to $\pm \infty$. These divergences, which behave as $-2 / \xi$ $(\xi>1)$ and $-2 /(1-\xi)(\xi<0)$, have been subtracted in Eq. (5). From the Ward identity, the integrated one-loop vertex correction is renormalized by the same terms. This ensures that the norm of the distributions is automatically preserved by the matching, i.e., $\int_{-\infty}^{\infty} d x h_{1}(x, \mu)=$ $\int_{-\infty}^{\infty} d x \tilde{h}_{1}\left(x, \mu, P_{3}\right)$ and $\int_{-\infty}^{\infty} d \xi \delta C\left(\xi, \frac{\xi \mu}{x P_{3}}\right)=1$ that holds for every value of $P_{3}$. Because particle number conservation is built inside the matching, the finite limits of integration imposed by the lattice data also conserve the norm.

\section{FINAL RESULTS}

A combination of the renormalization, matching procedure, and application of the TMCs allows the reconstruction of light-cone PDFs, which we present in this section. In Fig. 2, we show the effect of each step of this procedure for $P=\frac{10 \pi}{L}$; i.e., we start with the renormalized quasi-PDF $\left(\tilde{h}_{1}^{u-d}\right)$, apply matching $\left(h_{1}^{\prime u-d}\right)$, and finally include TMCs $\left(h_{1}^{u-d}\right)$, which leads to the final estimate of the transversity PDF. As can be seen, application of the matching shifts the peak of the distribution toward $x=0$ and increases it, as expected. We also find that TMCs are small, but non-negligible, and mostly affect the small- $x$ region.

Our final results are shown in Fig. 3 at a scale of $\sqrt{2} \mathrm{GeV}$ $\left(h_{1}^{u-d, \text { lattice }}\right)$. For clarity, we only show $P=\frac{10 \pi}{L}$, as the dependence on the nucleon momentum is small for most regions of $x$. We find that for the large and positive $x$ region the data at momentum $P=\frac{10 \pi}{L}$ have milder oscillatory behavior, an effect that originates from the use of finite

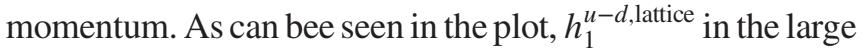

negative- $x$ nicely approach zero. For demonstration purposes, we include in the same plot phenomenological fits on SIDIS data [22] as well as SIDIS data constrained using lattice estimates of $g_{T}$ ("SIDIS + lattice") [22]. The statistical uncertainties of the lattice PDFs are strikingly smaller than the phenomenological fits of the SIDIS data. This also holds for the SIDIS + lattice data that have much smaller uncertainties than the unconstrained SIDIS values. The comparison favors the direct extraction of the transversity PDF using the quasi-PDFs method, in terms of uncertainties and reliability in the extraction. Using the data at $P=\frac{10 \pi}{L}$, we obtain $g_{T}=1.10$ (34) by integrating over $x$ within the interval $[-1,1]$. This value can be compared with the renormalized $M_{h_{1}}(P, 0)$ that gives a value $g_{T}=1.09(11)$, which is compatible with the aforementioned integration and with the extraction in Ref. [72]. Such an agreement is nontrivial since this work uses a stout-smeared operator as well as a generalized decomposition beyond the lab frame employed in earlier studies.

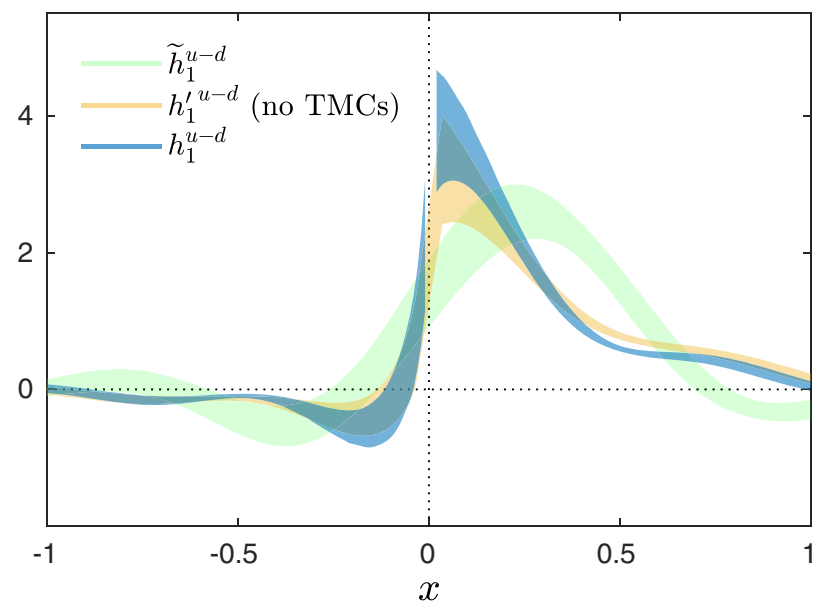

FIG. 2. Renormalized quasi-PDF, $\tilde{h}_{1}^{u-d}$ (green), PDF after matching, $h_{1}^{\prime u-d}$ (orange) and after TMCs, $h_{1}^{u-d}$ (blue), as a function of Bjorken- $x$ for $P=\frac{10 \pi}{L}$. 


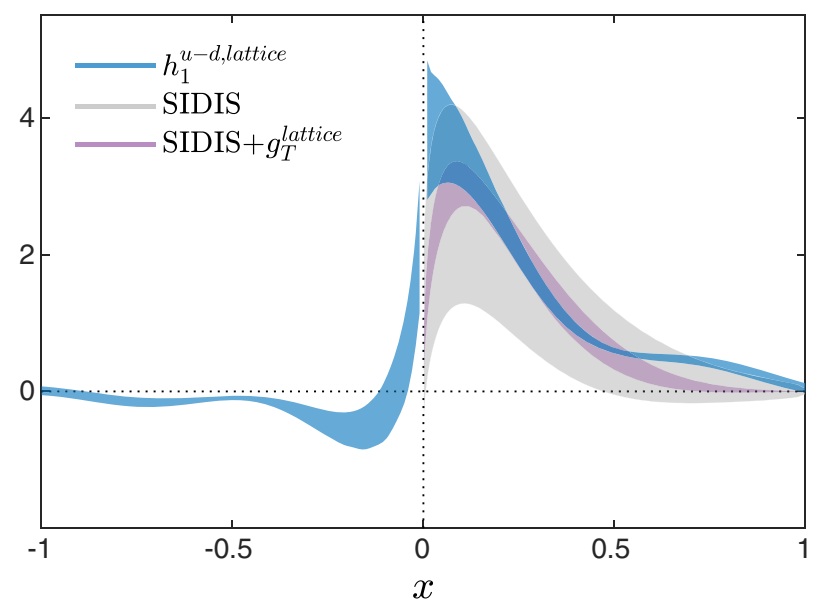

FIG. 3. Transversity PDF for $P=\frac{10 \pi}{L}$ (blue) as a function of Bjorken- $x$. The phenomenological fits have been obtained using SIDIS data (gray) [22] and SIDIS data constrained using $g_{T}^{\text {lattice }}$ (purple) [22].

\section{SUMMARY AND PROSPECTS}

This paper presents a state-of-the-art direct calculation of the transversity PDF for the isovector flavor combination. The novelty of this work is the improvement of the computation in all fronts, that is, simulations at physical quark masses [55,73], employment of a nonperturbative renormalization program [64], and application of a cutoff independent and renormalized matching between quasiPDFs and light-cone PDFs; the latter was developed in this work.

A number of careful investigations have been performed to study systematic uncertainties. We find that excited states are suppressed for a source-sink separation of $1.12 \mathrm{fm}$ and nucleon momentum up to $1.4 \mathrm{GeV}$. The twisted mass formulation has the advantage of automatic $\mathcal{O}(a)$ improvement, and calculation of the dispersion relation indicates small cutoff effects. Another investigation is the extraction of the tensor charge found to be $g_{T}=1.09(11)$, which is compatible with the dedicated study of Ref. [72], that is $g_{T}=$ $1.06(1)$ at $\mu=\sqrt{2} \mathrm{GeV}$. In addition, the renormalized $M_{h_{1}}(P, z)$ is completely independent of the stout smearing, which demonstrates the success of the renormalization program.

The great progress in the $a b$ initio calculation of PDFs is paving the way for further improvements. New computer architectures can lead to the extraction of reliable estimates for PDFs using higher nucleon momenta, that would presently require computational resources beyond what is currently available to eliminate excited states contamination. Development of new techniques to tackle the exponential increase of the statistical noise with respect to the sourcesink separation and higher momenta will be crucial for the quasi-PDFs calculations. If successful, future calculations at higher momenta will allow one to investigate higher-twist effects, while the continuum limit can be reliably taken using simulations with at least three values of the lattice spacing. Another direction that could potentially be improved is the calculation of renormalization functions, by utilizing the perturbative calculation of Ref. [42], and a technique to eliminate finite lattice spacing effects to order $\mathcal{O}\left(g^{2} a^{\infty}\right)$. The latter has successfully improved the renormalization functions of local operators [74].

The $a b$ initio calculation of the transversity PDF is a powerful demonstration of the predictability of lattice QCD. The final lattice results presented in Fig. 3 compare favorably to the phenomenological fits that carry very large uncertainties preventing any meaningful conclusions. Even though constraining the SIDIS data with lattice estimates on the tensor charge reduces the errors significantly, the overall uncertainties are still very large [22,23]. This reflects the large experimental difficulties to extract transversity PDFs, which, unlike the chiral-even distributions, can only be accessed if additional information from FFs is given. Thus, a computation from first principles is imperative, even more so when we compare it to its unpolarized and helicity counterparts, which can be directly probed in totally inclusive experiments. All the above point to the direction that a prediction of the transversity PDF directly from lattice QCD is a major breakthrough that opens new directions in understanding the immensely rich nucleon structure.

\section{ACKNOWLEDGMENTS}

We express gratitude to all members of ETMC for the pleasant collaboration. M.C. would like to thank ZeinEddine Meziani for many fruitful and inspiring discussions. F. S. thanks Nobuo Sato for helpful discussions and for providing the SIDIS phenomenological parametrizations. This work has received funding from the European Union's Horizon 2020 research and innovation programme under the Marie Skłodowska-Curie Grant No. 642069 (HPCLEAP). K. C. is supported by the National Science Centre SONATA BIS Grant No. 2016/22/E/ST2/00013. F. S. is funded by the Deutsche Forschungsgemeinschaft Project No. 392578569. M. C. acknowledges financial support by the U.S. Department of Energy, Office of Nuclear Physics, within the framework of the TMD Topical Collaboration, as well as by the National Science Foundation under Grant No. PHY-1714407. This research used resources of the Oak Ridge Leadership Computing Facility, which is a DOE Office of Science User Facility supported under Contract No. DE-AC05-00OR22725, Prometheus supercomputer at the Academic Computing Centre Cyfronet AGH in Cracow (Grant identification quasipdfs), Okeanos supercomputer at the Interdisciplinary Centre for Mathematical and Computational Modelling in Warsaw (Grants No. gb7017 and ga71-22), and Eagle supercomputer at the Poznan Supercomputing and Networking Center (Grant No. 346). 
[1] C. A. Aidala, S. D. Bass, D. Hasch, and G. K. Mallot, Rev. Mod. Phys. 85, 655 (2013).

[2] A. Accardi et al., Eur. Phys. J. C 76, 471 (2016).

[3] J. Gao, L. Harland-Lang, and J. Rojo, Phys. Rep. 742, 1 (2018).

[4] R. L. Jaffe and X.-D. Ji, Phys. Rev. Lett. 67, 552 (1991).

[5] J. L. Cortes, B. Pire, and J. P. Ralston, Z. Phys. C 55, 409 (1992).

[6] J. C. Collins, Nucl. Phys. B396, 161 (1993).

[7] R. Seidl et al. (Belle Collaboration), Phys. Rev. Lett. 96, 232002 (2006).

[8] R. Seidl et al. (Belle Collaboration), Phys. Rev. D 78, 032011 (2008); 86, 039905(E) (2012).

[9] I. Garzia (BABAR Collaboration), Proc. Sci., ICHEP2012 (2013) 272.

[10] A. Airapetian et al. (HERMES Collaboration), Phys. Lett. B 693, 11 (2010).

[11] M. Alekseev et al. (COMPASS Collaboration), Phys. Lett. B 673, 127 (2009).

[12] C. Adolph et al. (COMPASS Collaboration), Phys. Lett. B 744, 250 (2015).

[13] T. C. Rogers, Eur. Phys. J. A 52, 153 (2016).

[14] J. C. Collins, S. F. Heppelmann, and G. A. Ladinsky, Nucl. Phys. B420, 565 (1994).

[15] R. L. Jaffe, X.-m. Jin, and J. Tang, Phys. Rev. Lett. 80, 1166 (1998).

[16] A. Bacchetta, A. Courtoy, and M. Radici, Phys. Rev. Lett. 107, 012001 (2011).

[17] E. Moffat, W. Melnitchouk, T. C. Rogers, and N. Sato, Phys. Rev. D 95, 096008 (2017).

[18] M. Anselmino, M. Boglione, U. D’Alesio, A. Kotzinian, F. Murgia, A. Prokudin, and C. Turk, Phys. Rev. D 75, 054032 (2007).

[19] M. Anselmino, M. Boglione, U. D’Alesio, J. O. Gonzalez Hernandez, S. Melis, F. Murgia, and A. Prokudin, Phys. Rev. D 92, 114023 (2015).

[20] M. Radici, A. Courtoy, A. Bacchetta, and M. Guagnelli, J. High Energy Phys. 05 (2015) 123.

[21] Z.-B. Kang, A. Prokudin, P. Sun, and F. Yuan, Phys. Rev. D 93, 014009 (2016).

[22] H.-W. Lin, W. Melnitchouk, A. Prokudin, N. Sato, and H. Shows, Phys. Rev. Lett. 120, 152502 (2018).

[23] M. Radici and A. Bacchetta, Phys. Rev. Lett. 120, 192001 (2018).

[24] Z. Ye, N. Sato, K. Allada, T. Liu, J.-P. Chen, H. Gao, Z.-B. Kang, A. Prokudin, P. Sun, and F. Yuan, Phys. Lett. B 767, 91 (2017).

[25] D. Dubbers and M. G. Schmidt, Rev. Mod. Phys. 83, 1111 (2011).

[26] M. Cirelli, E. Del Nobile, and P. Panci, J. Cosmol. Astropart. Phys. 10 (2013) 019.

[27] T. Bhattacharya, V. Cirigliano, S. D. Cohen, A. Filipuzzi, M. Gonzalez-Alonso, M. L. Graesser, R. Gupta, and H.-W. Lin, Phys. Rev. D 85, 054512 (2012).

[28] A. Courtoy, S. Baeler, M. Gonzlez-Alonso, and S. Liuti, Phys. Rev. Lett. 115, 162001 (2015).

[29] J. Dudek et al., Eur. Phys. J. A 48, 187 (2012).

[30] A. Accardi et al., Eur. Phys. J. A 52, 268 (2016).

[31] E.-C. Aschenauer et al., Eur. Phys. J. A 53, 71 (2017).
[32] J.-W. Chen, S. D. Cohen, X. Ji, H.-W. Lin, and J.-H. Zhang, Nucl. Phys. B911, 246 (2016).

[33] C. Alexandrou, K. Cichy, M. Constantinou, K. Hadjiyiannakou, K. Jansen, F. Steffens, and C. Wiese, Phys. Rev. D 96, 014513 (2017).

[34] M. Constantinou, Proc. Sci., LATTICE2014 (2015) 001.

[35] C. Alexandrou, in Proceedings of the 45th International Symposium on Multiparticle Dynamics (ISMD 2015), Kreuth, Germany, 2015, Vol. 120, (2016), https://www .epj-conferences.org/articles/epjconf/abs/2016/15/contents/ contents.html.

[36] S. Syritsyn, Proc. Sci., LATTICE2013 (2014) 009.

[37] S. Capitani, M. Della Morte, D. Djukanovic, G. M. von Hippel, J. Hua, B. Jger, P. M. Junnarkar, H. B. Meyer, T. D. Rae, and H. Wittig, arXiv:1705.06186.

[38] C. C. Chang et al., Nature (London) 558, 91 (2018).

[39] R. Gupta, Y.-C. Jang, B. Yoon, H.-W. Lin, V. Cirigliano, and T. Bhattacharya, Phys. Rev. D 98, 034503 (2018).

[40] K.-I. Ishikawa, Y. Kuramashi, S. Sasaki, N. Tsukamoto, A. Ukawa, and T. Yamazaki, Phys. Rev. D 98, 074510 (2018).

[41] X. Ji, Phys. Rev. Lett. 110, 262002 (2013); C. Müller, J. Bourassa, and A. Blais, Phys. Rev. B 88, 235401 (2013).

[42] M. Constantinou and H. Panagopoulos, Phys. Rev. D 96, 054506 (2017).

[43] X. Ji, Sci. China Phys. Mech. Astron. 57, 1407 (2014).

[44] C. Alexandrou, K. Cichy, M. Constantinou, K. Jansen, A. Scapellato, and F. Steffens, Phys. Rev. Lett. 121, 112001 (2018).

[45] J.-W. Chen, L. Jin, H.-W. Lin, Y.-S. Liu, Y.-B. Yang, J.-H. Zhang, and Y. Zhao, arXiv:1803.04393.

[46] A. V. Radyushkin, Phys. Rev. D 96, 034025 (2017).

[47] K. Orginos, A. Radyushkin, J. Karpie, and S. Zafeiropoulos, Phys. Rev. D 96, 094503 (2017).

[48] Y.-Q. Ma and J.-W. Qiu, Phys. Rev. Lett. 120, 022003 (2018).

[49] A. Radyushkin, arXiv:1807.07509.

[50] J. Karpie, K. Orginos, and S. Zafeiropoulos, arXiv:1807 .10933.

[51] Y. Iwasaki, arXiv:1111.7054.

[52] A. Abdel-Rehim et al., Proc. Sci., LATTICE2013 (2014) 264.

[53] R. Frezzotti and G. C. Rossi, J. High Energy Phys. 08 (2004) 007.

[54] B. Sheikholeslami and R. Wohlert, Nucl. Phys. B259, 572 (1985).

[55] A. Abdel-Rehim et al. (ETM Collaboration), Phys. Rev. D 95, 094515 (2017).

[56] W.-C. Chang and J.-C. Peng, Prog. Part. Nucl. Phys. 79, 95 (2014).

[57] S. Gusken, U. Low, K. H. Mutter, R. Sommer, A. Patel, and K. Schilling, Phys. Lett. B 227, 266 (1989).

[58] C. Alexandrou, S. Gusken, F. Jegerlehner, K. Schilling, and R. Sommer, Nucl. Phys. B414, 815 (1994).

[59] M. Albanese et al. (APE Collaboration), Phys. Lett. B 192, 163 (1987).

[60] G. Martinelli and C. T. Sachrajda, Nucl. Phys. B316, 355 (1989).

[61] G. S. Bali, B. Lang, B. U. Musch, and A. Schäfer, Phys. Rev. D 93, 094515 (2016). 
[62] G. S. Bali, S. Collins, and A. Schafer, Comput. Phys. Commun. 181, 1570 (2010).

[63] C. Morningstar and M. J. Peardon, Phys. Rev. D 69, 054501 (2004).

[64] C. Alexandrou, K. Cichy, M. Constantinou, K. Hadjiyiannakou, K. Jansen, H. Panagopoulos, and F. Steffens, Nucl. Phys. B923, 394 (2017).

[65] J. Green, K. Jansen, and F. Steffens, Phys. Rev. Lett. 121, 022004 (2018).

[66] X. Xiong, X. Ji, J.-H. Zhang, and Y. Zhao, Phys. Rev. D 90, 014051 (2014).

[67] C. Alexandrou, K. Cichy, V. Drach, E. Garcia-Ramos, K. Hadjiyiannakou, K. Jansen, F. Steffens, and C. Wiese, Phys. Rev. D 92, 014502 (2015).
[68] J.-W. Chen, X. Ji, and J.-H. Zhang, Nucl. Phys. B915, 1 (2017).

[69] W. Wang, S. Zhao, and R. Zhu, Eur. Phys. J. C 78, 147 (2018).

[70] I. W. Stewart and Y. Zhao, Phys. Rev. D 97, 054512 (2018).

[71] T. Izubuchi, X. Ji, L. Jin, I. W. Stewart, and Y. Zhao, Phys. Rev. D 98, 056004 (2018).

[72] C. Alexandrou et al., Phys. Rev. D 95, 114514 (2017).

[73] A. Abdel-Rehim et al., Phys. Rev. D 92, 114513 (2015); 93, 039904(E) (2016).

[74] C. Alexandrou, M. Constantinou, and H. Panagopoulos (ETM Collaboration), Phys. Rev. D 95, 034505 (2017). 